\title{
Can Femoral Rotation Be Localized and Quantified Using Standard CT Measures?
}

\author{
Andrew G. Georgiadis MD, Daniel S. Siegal MD, \\ Courtney E. Scher DO, Ira Zaltz MD
}

Published online: 22 October 2014

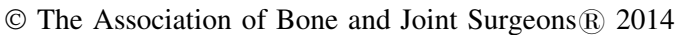

\begin{abstract}
Background The terms "femoral anteversion" and "femoral torsion" have often been used interchangeably in the orthopaedic literature, yet they represent distinct anatomical entities. Anteversion refers to anterior tilt of the femoral neck, whereas torsion describes rotation of the femoral shaft. Together, these and other transverse plane differences describe what may be considered rotational deformities of the femur. Assessment of femoral rotation is now routinely measured by multiple axial CT methods. The most widely used radiographic technique (in which only
\end{abstract}

Each author certifies that he or she, or a member of his or her immediate family, has no funding or commercial associations (eg, consultancies, stock ownership, equity interest, patent/licensing arrangements, etc) that might pose a conflict of interest in connection with the submitted article.

All ICMJE Conflict of Interest Forms for authors and Clinical Orthopaedics and Related Research ${ }^{\mathbb{R}}$ editors and board members are on file with the publication and can be viewed on request.

Clinical Orthopaedics and Related Research ${ }^{\mathbb{R}}$ neither advocates nor endorses the use of any treatment, drug, or device. Readers are encouraged to always seek additional information, including FDA-approval status, of any drug or device prior to clinical use. This work was performed at Henry Ford Hospital, Detroit, MI, USA.

A. G. Georgiadis, I. Zaltz

Department of Orthopaedic Surgery, Henry Ford Hospital,

Detroit, MI, USA

D. S. Siegal, C. E. Scher

Department of Radiology, Henry Ford Hospital, Detroit, MI, USA

\section{Zaltz (ه)}

Department of Orthopaedic Surgery, William Beaumont Hospital, 3535 West Thirteen Mile Road, Suite 744, Royal Oak, MI 48073, USA

e-mail: zaltzira@gmail.com two CT-derived axes are made, one through the femoral neck and one at the distal femoral condyles) may not accurately quantify proximal femoral anatomy nor allow identification of the anatomic locus of rotation.

Questions/purposes (1) What CT methodology (a twoaxis CT-derived technique, a three-axis technique adding an intertrochanteric axis-the "Kim method," or a volumetric three-dimensional reconstruction of the proximal femur) most accurately quantifies transverse plane femoral morphology; (2) localizes those deformities; and (3) is most reproducible across different observers?

Methods We constructed a high-definition femoral sawbones model in which osteotomies were performed at either the intertrochanteric region or femoral shaft. Transverse plane deformity was randomly introduced and CTderived rotational profiles were constructed using three different CT methods. Accuracy and consistency of measurements of femoral rotation were calculated using p values and Fisher's exact test and intraclass correlation coefficients (ICCs).

Results All three CT methodologies accurately quantified overall transverse plane rotation (mean differences $0.69^{\circ} \pm 3.88^{\circ}, 0.69^{\circ} \pm 3.88^{\circ}$, and $-1.09^{\circ} \pm 4.44^{\circ}$ for the two-plane, Kim, and volumetric methods, respectively). However, use of a single neck and single distal femoral axis does not reliably identify the anatomic locus of rotation, whereas the Kim and volumetric methods do $(\mathrm{p}<0.0001)$. All three methods were highly reproducible between observers (ICCs of 0.9569, 0.9569, and 0.9359 for the traditional two-plane, Kim, and volumetric methods, respectively).

Conclusions Only the Kim and volumetric methods can identify the anatomic location of transverse plane rotation and we recommend using one of the two techniques. Accurate anatomic localization of transverse plane rotation 
enables using precise anatomic terminology ("femoral torsion" versus "femoral [ante]version").

Clinical Relevance Current descriptions and treatment of femoral rotational deformities do not discriminate the location of rotation. The transverse plane femoral rotation requires a precise definition based on its anatomic location to maintain consistent communication between clinicians, because version of the neck and torsion of the shaft may have different treatment.

\section{Introduction}

The terms "femoral anteversion" and "femoral torsion" have often been used interchangeably in the orthopaedic literature, yet they represent distinct anatomical entities. Anteversion refers to anterior tilt of the femoral neck, whereas torsion describes rotation of the femoral shaft. Before the widespread use of CT, the location of rotation was ignored. Early authors stated that neck anteversion and shaft torsion could not be distinguished roentgenologically, deeming such discrimination to be of "no practical importance" [2]. With CT, however, more accurate threedimensional evaluation became possible. Currently, despite radiologic advances for characterizing axial femoral morphology, the terms "anteversion" and "torsion" are still used interchangeably in the primary literature $[4,6,16,17]$. Treatment of femoral neck deformity may be different than diaphyseal deformity, and clinicians should use terms that differentiate these morphologies.

Assessment of femoral rotation by CT is now routinely measured by several methods. However, the most widely used radiographic technique (in which only two CTderived axes are made, one through the femoral neck and one at the distal femoral condyles) may neither accurately quantify proximal femoral anatomy nor identify the center of rotation. A second technique, popularized by Kim, adds an intertrochanteric axis, and thus provides three axes for measurement. A final approach uses three-dimensional volumetric reconstruction of the proximal femur.

We therefore sought to identify what CT methodology (the traditional method, Kim method, or volumetric method) (1) most accurately quantifies transverse-plane femoral deformities; (2) localizes those deformities; and (3) is most reproducible across different observers.

\section{Materials and Methods}

We designed a sawbones study in which femoral rotational abnormalities could be readily simulated. Medium-sized, left, high-resolution femurs of standardized anteversion (Pacific Research Laboratories, Vashon, WA, USA) were used. Simple transverse osteotomies were performed with an oscillating saw in either the subtrochanteric region (ST), $4 \mathrm{~cm}$ distal to the inferior aspect of the lesser trochanter, or the intertrochanteric region (IT), $2 \mathrm{~cm}$ proximal to the superior aspect of the lesser trochanter. These levels were chosen because of the uniform thickness of the sawbones "cortex" (ST level) to simulate diaphyseal rotation, and IT osteotomies are generally performed within $2 \mathrm{~cm}$ of the lesser trochanter and an osteotomy further cephalad would have involved the femoral neck.

Femora were rotated in the diaphyseal or intertrochanteric region around their geometric centroid as defined by Murphy et al. [10]. The distal fragment of all femora was internally rotated relative to the proximal fragment. Each sample was marked circumferentially in $1^{\circ}$ increments and then rotated a random value between $0^{\circ}$ and $45^{\circ}$ according to a sequential randomization schedule developed by an independent statistician (AT). The femora were secured with a radiolucent adhesive (Fig. 1). Rotational values were selected to mimic a range of values of femoral deformity that are encountered clinically [14]. The full lengths of all femora were then subjected to axial CT. All scans were performed on a GE Discovery 64-detector scanner (General Electric, Fairfield, CT, USA) with slice thickness $0.6 \mathrm{~mm}, 120 \mathrm{KvP}, 312 \mathrm{~mA}$, and $500 \mathrm{~ms}$. Any axial images on which the osteotomy was visible were removed.

Measurements of femoral rotation were performed by each of three methods by two fellowship-trained musculoskeletal radiologists (DSS, CES) blinded to the study design. The "traditional" method described by Weiner et al. [18] incorporated the femoral neck axis from a single axial image and a single posterior condylar axis. The posterior condylar axis provides the most accurate and reproducible distal reference point [10]. The method of Kim et al. [7] involved these axes and a third "intertrochanteric" best-fit line in which the greater and lesser trochanters were bisected as determined from two axial images, allowing qualitative localization of rotation to either the supra- or infratrochanteric region. The third method incorporated three-dimensional (3-D) volumetric reconstruction of the proximal femur for a true cervical axis (Philips iSite Advanced Visualization Suite, Version 7.4.6.500, Amsterdam, The Netherlands) while the posterior condylar axis was identically obtained (Fig. 2). A repeat set of measurements of all femora was separated by an interval of 6 months. The observers were also asked to identify the location of rotation (IT versus ST region) in each sample.

An a priori sample size estimation analysis was performed under the following assumptions: a paired t-test 


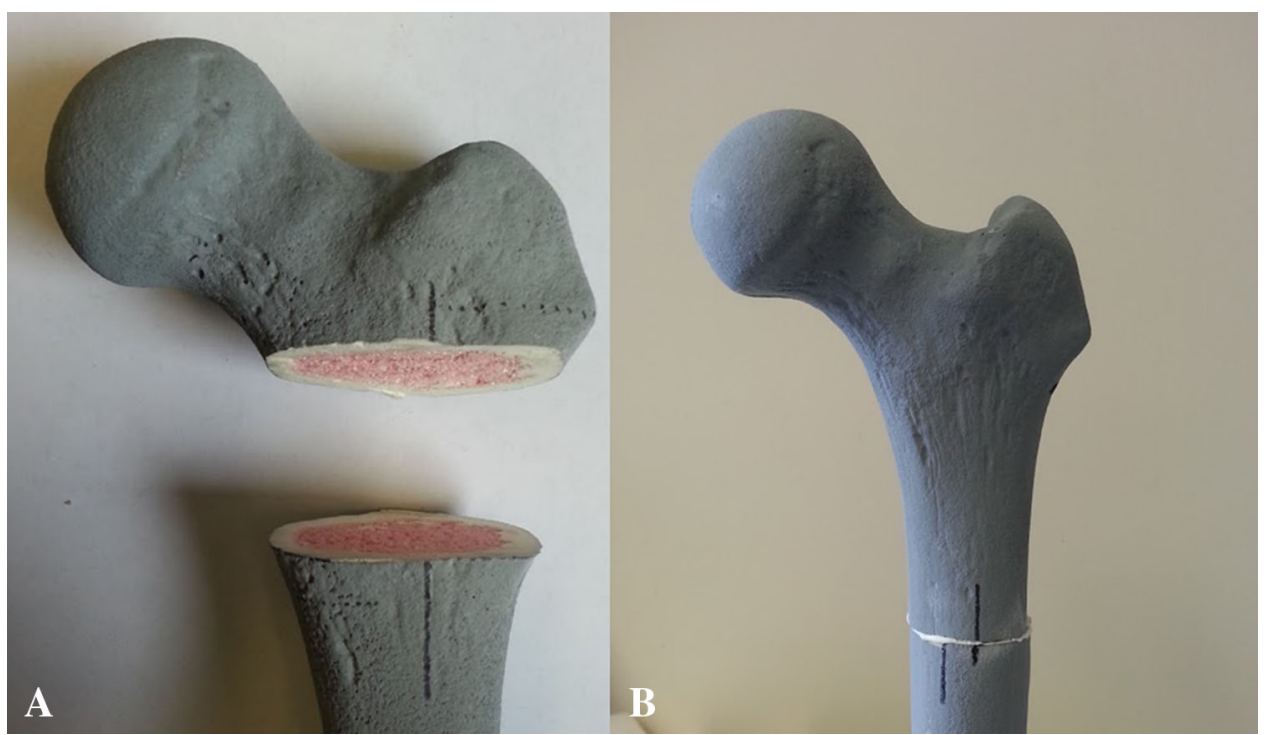

Fig. 1A-B (A) Pictured is a high-definition sawbones femur with an intertrochanteric osteotomy. (B) Pictured is a femur with a subtrochanteric osteotomy after rotation and security with radiolucent adhesive.

design with a correlation between the two sets of observations of 0.94 , power set to 0.80 , and alpha set at 0.05 for a two-sided test. The sample size estimation investigated the number of pairs of observations that would be necessary to detect a significant effect given: (1) a difference in the rotational degree of either $5^{\circ}$ or $10^{\circ}$; and (2) assuming a $\mathrm{SD}$ of $5^{\circ}$, all consistent with detectable differences in previous CT-based cadaveric femoral rotation studies [10]. A minimum of four samples from each osteotomy type (ST or IT) was statistically necessary, and we conducted our study with eight samples for each osteotomy type (16 total).

All analyses were performed using SAS 9.2 (Cary, NC, USA). The level of statistical significance was set at $p \leq 0.05$. Fisher's exact tests were used to compare the frequency of correctly identifying the location of the rotation by each of the three methods. The percentage of correct identifications by each method was compared for each observer and for both observers combined.

The intraclass correlation coefficient (ICC) was calculated to look at intermethod agreement and interrater agreement. It is a measure of reliability of the outcomes and indicates whether there is strong agreement between continuous values of rotation (true versus traditional, true versus Kim, true versus volumetric, Observer 1 versus Observer 2). An ICC of 0.81 to 1.00 represents "almost perfect agreement" [8]. Agreement across time was assessed for both location and degree of rotation. Similar to previous analyses, agreement for identifying the location of rotation (IT versus ST) was calculated using Cohen's kappa.

\section{Results}

With the numbers available, no differences were identified among the traditional, Kim, and volumetric CT techniques in detecting the total amount of transverse-plane femoral deformity in this model (Table 1). Specifically, the traditional and Kim methods averaged $-1^{\circ} \pm 4^{\circ}$ from true rotation (range, $-7^{\circ}$ to $7^{\circ} ; \mathrm{p}=0.4895$ ), and the volumetric method averaged rotation $1^{\circ} \pm 4^{\circ}$ from true rotation (range, $-7^{\circ}$ to $7^{\circ} ; \mathrm{p}=0.3404$ ).

The Kim and volumetric techniques were superior to the traditional technique in terms of localizing the deformity, and with the numbers available, we identified no differences in performance between the Kim and volumetric techniques. With traditional measurements using two axial images (single femoral neck axis, single posterior condylar axis), the correct site of torsion was identified $47 \%$ of the time compared with $100 \%$ with the trochanteric method of Kim and $100 \%$ with volumetric analysis $(\mathrm{p}<0.001$; Table 2).

There was near perfect agreement between observers in quantifying rotation by all three methods (Table 1). At index measurement, the ICCs of the traditional, Kim, and volumetric methods were $0.9569,0.9569$, and 0.9359 , respectively. The interrater reliability of the angular measurements persisted when a second set of measurements was taken 6 months later (Table 3). After allowing 6 months to elapse, the ICCs of the traditional, Kim, and volumetric methods were $0.9808,0.9807$, and 0.9353 , respectively. Localizing the site of femoral rotation was inconsistent between observers by the traditional method 

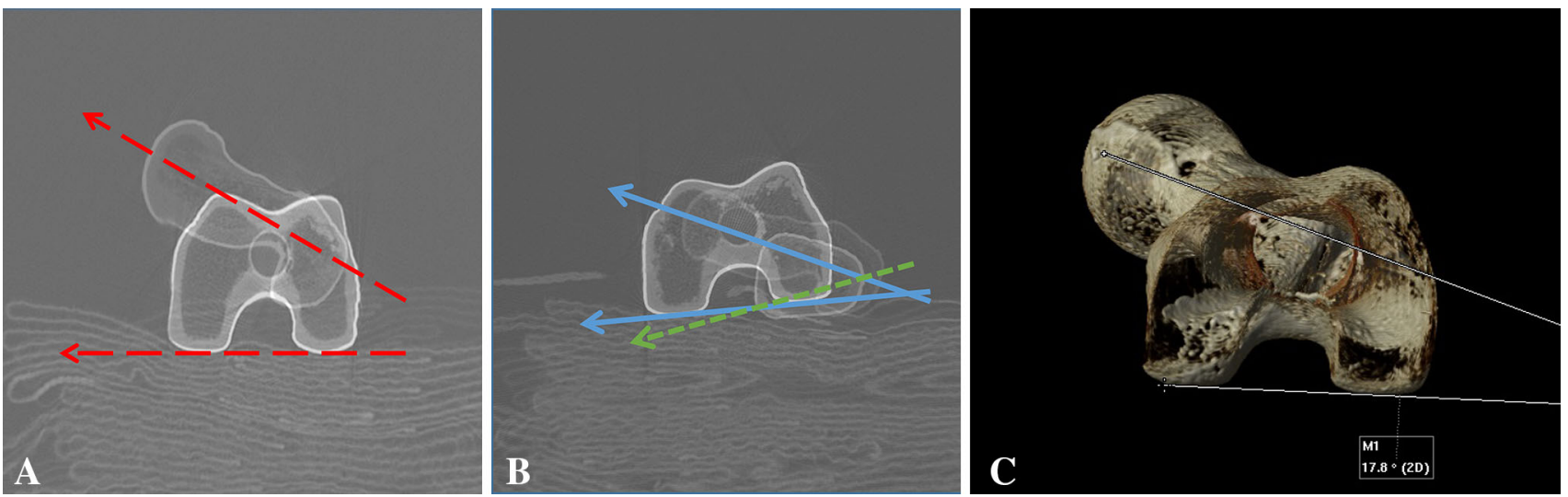

Fig. 2A-C (A) An example of the traditional method is shown, in which femoral rotation is assessed by a single cervical axis and the posterior condylar axis. (B) An example of the Kim method is shown, whereby single cervical and posterior condylar axes are drawn. An intertrochanteric line (dashed) bisecting the greater and lesser trochanters allows evaluation of torsional contribution from the neck versus the diaphysis. The intertrochanteric line is drawn by finding the

$(\kappa=0.375)$. In contrast, the Kim and volumetric techniques showed almost perfect kappa values $(\kappa=1.0)$, indicating that every time one observer indicated a location, the other observer indicated that same location (Table 4).

\section{Discussion}

Femoral rotation has been variably termed "anteversion" or "torsion" regardless of the site of rotation. Transverseplane femoral profiles are currently measured using axial CT, and the often-used method of a single cervical axis has been suggested to introduce a measurement error. This study aimed to determine which of the existing techniques of CT-derived transverse plane femoral profiles is most accurate and reproducible. We found that a volumetric 3-D reconstruction of the proximal femur and a three-axis technique adding an intertrochanteric axis (the "Kim method") were more accurate than a two-axis technique at (1) quantifying transverse plane femoral morphology; (2) localizing those deformities; and (3) were more reproducible among different observers.

This study has a number of limitations. First, it is a sawbones model and not a clinical study. Although a sawbones model allows complete investigator control of rotation, the heterogeneity of rotational deformity in vivo cannot be mimicked. Femoral rotational deformity can stem from multiple loci along the femoral length. Furthermore, some femora in the clinical setting may be retroverted and deformity may stem from the neck or epiphysis, which was not simulated in this investigation. Volumetric transverse plane profiles were constructed with midpoint of the greater trochanter on a single axial image and the midpoint of the lesser trochanter on a single axial image and connecting those points. In this femur, the torsion is emanating from above the lesser trochanter. (C) A representative volumetric 3-D reconstruction of transverse plane femoral morphology from a true cervical axis is shown. This axis is generated from radiological software as a "true" composite neck axis.

complex software not widely available to all surgeons and radiologists; hence, the inclusion of the Kim method [7], which provides a straightforward technique for rotational localization available with any CT scanner. It also provides for quantification of a mixed supra- and infratrochanteric rotational deformity. This method can itself be subject to measurement error, because the profiles of the greater and lesser trochanters may not be readily identifiable in a deformed femur. One other limitation of the study is the anatomic variation of the location of the lesser trochanter in vivo. How this affects this study and the calculation and localization of rotation is not currently fully understood, although it is assumed that the lesser trochanter is generally consistently located on the posteromedial femur. It could affect and limit the applicability of the Kim method if in vivo variation exists. Although we examined eight femora in each group (IT and ST), some differences of modest size could be missed because of Type II error, although a prestudy power analysis confirmed adequate sample size.

In our study, we found equivalent accuracy for each measurement technique in quantifying femoral rotation, which was unanticipated. This was an unexpected finding because Murphy et al. [10] found significant underestimation of rotation using single neck axis cuts in their cadaveric CT investigation. Before the advent of CT, both direct [5] and indirect [2, 11-13, 15, 17] plain radiographic techniques were used to characterize femoral rotation. CT allowed for direct superimposition of a single neck and condylar axis, which remains the basis for most measurements to this day [18]. However, the femoral neck is neither uniplanar nor cylindrical and can be best approximated by a series of ellipses that create a unique composite 
Table 1. Assessment of femoral rotation with three distinct CTderived methods

\begin{tabular}{ccccc}
\hline Comparison & $\begin{array}{l}\text { Mean } \\
\text { difference } \pm \text { SD } \\
\text { (range) }\end{array}$ & $\begin{array}{l}\text { p value for } \\
\text { paired t-test* }\end{array}$ & ICC & Agreement \\
\hline $\begin{array}{c}\text { True versus } \\
\text { traditional }\end{array}$ & $1 \pm 4(-7$ to 7$)$ & 0.4895 & 0.9569 & $\begin{array}{c}\text { Almost } \\
\text { perfect }\end{array}$ \\
$\begin{array}{c}\text { True versus } \\
\text { Kim }\end{array}$ & $1 \pm 4(-7$ to 7$)$ & 0.4895 & 0.9569 & $\begin{array}{c}\text { Almost } \\
\text { perfect }\end{array}$ \\
$\begin{array}{c}\text { True versus } \\
\text { volumetric }\end{array}$ & $-1 \pm 4(-7$ to 7$)$ & 0.3404 & 0.9395 & $\begin{array}{c}\text { Almost } \\
\text { perfect }\end{array}$ \\
\hline
\end{tabular}

The mean difference in degrees (calculated as method-true) is given for each comparison. The ICC for each comparison indicates almost perfect agreement between the true and measured values by all methods. These results suggest all examined techniques were reliable; *this is not the $\mathrm{p}$ value for the ICC; ICC $=$ intraclass correlation coefficient.

Table 2. Correct localization of femoral rotation

\begin{tabular}{|c|c|c|c|c|}
\hline \multirow[t]{2}{*}{ Observer } & \multirow[t]{2}{*}{ Method } & \multicolumn{2}{|c|}{$\begin{array}{l}\text { Did the observer } \\
\text { correctly identify } \\
\text { the location? }\end{array}$} & \multirow[t]{2}{*}{$\mathrm{p}$ value } \\
\hline & & Yes & No & \\
\hline \multirow[t]{3}{*}{ Observer 1} & Traditional & $10(63)$ & $6(38)$ & \multirow[t]{3}{*}{0.002} \\
\hline & Kim & $16(100)$ & $0(0)$ & \\
\hline & Volumetric & $16(100)$ & $0(0)$ & \\
\hline \multirow[t]{3}{*}{ Observer 2} & Traditional & $5(31)$ & $11(69)$ & \multirow[t]{3}{*}{$<0.001$} \\
\hline & Kim & $16(100)$ & $0(0)$ & \\
\hline & Volumetric & $16(100)$ & $0(0)$ & \\
\hline \multirow{3}{*}{$\begin{array}{l}\text { Overall (both observers } \\
\text { combined) }\end{array}$} & Traditional & $15(47)$ & $17(53)$ & \multirow[t]{3}{*}{$<0.001$} \\
\hline & Kim & $32(100)$ & $0(0)$ & \\
\hline & Volumetric & $32(100)$ & $0(0)$ & \\
\hline
\end{tabular}

Results are reported in sample numbers and percentage. When using the traditional method, Observer 1 and Observer 2 were less likely to correctly identify the location of rotation as compared with the Kim and volumetric methods. The results persisted when both observers were combined. Using the Kim and volumetric methods, the observers correctly identified the location $100 \%$ of the time, whereas doing so only $47 \%$ of the time using the traditional method.

neck axis, which deviates from the shaft axis by an average $5 \mathrm{~mm}$ and the center of the femoral head by $2.5 \mathrm{~mm}$ [3]. As a result of this complex proximal femoral anatomy, some authors have suggested that the traditional methods of axial CT-derived femoral profiles are inaccurate and underestimate true anatomic measurements. The clinical significance of this measurement error in CT-derived profiles is not known. In this investigation, transverse-plane rotational profiles were quantified equally well by the traditional, Kim, and 3-D/volumetric methods.

The traditional method of a uniplanar neck axis did not enable anatomic localization of the site of rotation, whereas the Kim and volumetric methods did. In this study, the
Table 3. Accuracy and interrater reliability of measurements over time

\begin{tabular}{lcllc}
\hline Comparison & $\begin{array}{l}\text { Mean difference } \\
\left({ }^{\circ} \pm \text { SD (range) }\right.\end{array}$ & $\begin{array}{l}\text { p value } \\
\text { for } \\
\text { paired } \\
\text { t-test* }\end{array}$ & ICC & Agreement \\
\hline $\begin{array}{c}\text { Traditional, Time } \\
1 \text { versus Time 2 }\end{array}$ & $1 \pm 2(-3$ to 5) & 0.0310 & 0.9808 & $\begin{array}{c}\text { Almost } \\
\text { perfect }\end{array}$ \\
$\begin{array}{c}\text { Kim, Time 1 } \\
\text { versus Time 2 }\end{array}$ & $1 \pm 2(-3$ to 5) & 0.0321 & 0.9807 & $\begin{array}{c}\text { Almost } \\
\text { perfect }\end{array}$ \\
$\begin{array}{c}\text { Volumetric, Time } \\
1 \text { versus Time 2 }\end{array}$ & $0^{*} \pm 2(-5$ to 5) & 0.8064 & 0.9353 & $\begin{array}{c}\text { Almost } \\
\text { perfect }\end{array}$ \\
\hline
\end{tabular}

The agreement and reliability between Time 1 and Time 2 for each method are displayed. There was almost perfect agreement between observers for each measurement technique as indicated by high ICCs. The traditional and Kim methods had higher rotational measurements at Time 2 (average $+1^{\circ}, \mathrm{p}=0.0310-0.0321$ ) but this likely does not represent a clinically significant finding; *raw volumetric measurements were within an average of $0.09^{\circ}$ between observation Time 1 and Time 2; ICC $=$ intraclass correlation coefficient.

Table 4. Interrater agreement on the location of femoral rotation

\begin{tabular}{lll}
\hline Observer 1 versus Observer 2 & Kappa & Agreement \\
\hline Traditional & 0.3750 & Fair agreement \\
Kim & 1.0 & (Almost) perfect agreement \\
Volumetric & 1.0 & (Almost) perfect agreement
\end{tabular}

Cohen's kappa agreement coefficients for each of three measurement techniques in identifying the site of femoral rotation. Using the traditional method, there was relatively poor interrater agreement. This indicates that there was a weak association between when one observer identified the anatomic locus of rotation as IT or ST and the second observer identifying the same location. Alternatively, the Kim and volumetric techniques showed near perfect kappas, indicating that every time one observer identified a location, the other observer identified the same location; IT = intertrochanteric; ST = subtrochanteric.

addition of the intertrochanteric CT axis described by Kim to the traditional method allowed differentiation of "supratrochanteric" and "infratrochanteric" femoral rotation and was the basis for localizing an otherwise hidden osteotomy. This added CT axis enabled the differentiation of femoral "anteversion" and "torsion" or the relative contributions of each to a mixed rotational profile. Without it, our observers identified the location of femoral rotation only $47 \%$ of the time and the two observers had poor interrater agreement in the instances in which they were correct. In sum, the site of rotation could only be identified when using a technique that considered anatomic details between the femoral neck and distal condyles (ie, the Kim or volumetric methods).

All three CT methodologies had high interrater reliability. Although other authors have successfully used two- 
dimensional (2-D) CT to obtain accurate rotational profiles despite complex femoral neck anatomy [1,3,9], it has been noted that variability in the traditional method is minimized with more experienced observers (attending physicians versus residents) and the use of predefined cut levels [9]. In our investigation, both the traditional and Kim methods (2-D) provided excellent inter- and intraobserver reliability as did the volumetric (3-D) technique.

Despite the complex 3-D anatomy of the proximal femur, we have demonstrated that the overall rotational profile can be accurately and reproducibly measured using traditional 2$\mathrm{D}$ techniques, even with a single neck axis. However, the locus of rotation (neck versus shaft) cannot be identified without, at minimum, some evaluation of the intertrochanteric region. In the absence of software providing 3-D volumetric analysis of the proximal femur, an intertrochanteric CT axis localizing the trochanters can differentiate rotation occurring proximal to the lesser trochanter from rotation occurring distal to the lesser trochanter. As the biomechanical role of femoral rotation on hip mechanics becomes increasingly understood, it is important to both accurately measure and localize the site of femoral rotation. Furthermore, using unambiguous terminology that reflects the site of rotation will facilitate discussions between practitioners and investigators. In the future, we suggest that when referring to transverse plane rotation of the femur and its surgical treatment that "version" be used for rotation localized proximal to the lesser trochanter and "torsion" be used for rotation localized distal to the lesser trochanter.

Acknowledgments We thank Clifford M. Les DVM, PhD, for his contribution to the a priori sample size analysis and Andrew Taylor MA, for his post hoc statistical analysis.

\section{References}

1. Abel MF, Sutherland DH, Wenger DR, Mubarak SJ. Evaluation of CT scans and 3-D reformatted images for quantitative assessment of the hip. J Pediatr Orthop. 1994;14:48-53.
2. Billing L. Roentgen examination of the proximal femur end in children and adolescents; a standardized technique also suitable for determination of the collum-, anteversion-, and epiphyseal angles; a study of slipped epiphysis and coxa plana. Acta Radiol Suppl. 1954;110:1-80.

3. Bonneau N, Libourel PA, Simonis C, Puymerail L, Baylac M, Tardieu C, Gagey O. A three-dimensional axis for the study of femoral neck orientation. J Anat. 2012;221:465-476.

4. Botser IB, Ozoude GC, Martin DE, Siddiqi AJ, Kuppuswami S, Domb BG. Femoral anteversion in the hip: comparison of measurement by computed tomography, magnetic resonance imaging, and physical examination. Arthroscopy. 2012;28:619-627.

5. Budin E, Chandler E. Measurement of femoral neck anteversion by a direct method. Radiology. 1957;69:209-213.

6. Kate BR. Anteversion versus torsion of the femoral neck. Acta Anat. 1976;94:457-463.

7. Kim HY, Lee SK, Lee NK, Choy WS. An anatomical measurement of medial femoral torsion. $J$ Pediatr Orthop $B$. 2012;21:552-557.

8. Landis JR, Koch GG. The measurement of observer agreement for categorical data. Biometrics. 1977;33:159-174.

9. Liodakis E, Doxastaki I, Chu K, Krettek C, Gaulke R, Citak M, Kenawey M. Reliability of the assessment of lower limb torsion using computed tomography: analysis of five different techniques. Skeletal Radiol. 2012;41:305-311.

10. Murphy SB, Simon SR, Kijewski PK, Wilkinson RH, Griscom NT. Femoral anteversion. J Bone Joint Surg Am. 1987;69:11691176.

11. Rippstein J. [Determination of the antetorsion of the femur neck by means of two x-ray pictures] [in German]. Z Orthop Ihre Grenzgeb. 1955;86:345-360.

12. Rogers SP. A method for determining the angle of torsion of the neck of the femur. J Bone Joint Surg. 1931;13:821-824.

13. Rogers SP. Observations on torsion of the femur. $J$ Bone Joint Surg. 1934;16:284-289.

14. Ruwe PA, Wright J, Randall RL, Lynch JK, Jokl P, McCarthy S. Can MR imaging effectively replace diagnostic arthroscopy? Radiology. 1992;183:335-339.

15. Ryder CT, Crane L. Measuring femoral anteversion; the problem and a method. J Bone Joint Surg Am. 1953;35:321-328.

16. Sangeux M, Mahy J, Graham HK. Do physical examination and CT-scan measures of femoral neck anteversion and tibial torsion relate to each other? Gait Posture. 2014;39:12-16.

17. Shands AR Jr, Steele MK. Torsion of the femur; a follow-up report on the use of the Dunlap method for its determination. J Bone Joint Surg Am. 1958;40:803-816.

18. Weiner DS, Cook AJ, Hoyt WA, Jr., Oravec CE. Computed tomography in the measurement of femoral anteversion. Orthopedics. 1978;1:299-306. 